\title{
ESTUDIO COMPUTACIONAL DE LA REACTIVIDAD COMPARADA DE LOS ANIONES TIOACETATO Y TIOBENZOATO FRENTE AL BIS-(2,4-DINITROFENIL)-ÉTER Y AL BIS-(2,4- DINITROFENIL)-SULFURO
}

\author{
Jorge Cappetta $^{\mathrm{a}^{*}}$ César Alfonso Micheli ${ }^{\mathrm{a}}$
}

\begin{abstract}
RESUMEN
Mediante metodologías computacionales, empleando los programas Mecánica Molecular y PM3 (semiempírico) se estudian comparativamente la sustitución nucleofílica aromática bimolecular de cuatro reacciones de las especies del título de este trabajo. Los resultados obtenidos se comparan con el comportamiento cinético observado experimentalmente. El análisis energético aproxima las energías libres de activación correspondientes y permite establecer: una escala de reactividad de las reacciones objeto de estudio, como así también de la nucleofilia y electrofilia relativas de los reactivos involucrados en la primera etapa.

Además, los datos energéticos obtenidos se cotejan con determinaciones simuladas de una parte de los efectos de impedimento estérico relacionados con reactivos e intermedios investigados.

Los resultados de estas comparaciones se correlacionan e interpretan haciendo uso de los criterios mecanísticos básicos de la química orgánica.

Palabras clave: Sustitución nucleofílica aromática bimolecular, compuestos orgánicos del azufre bivalente, química orgánica computacional, reactividad comparada, modelización de la interacción nucleófilos-sustratos, impedimento estérico.
\end{abstract}

\begin{abstract}
Though the use of informatics programs, at level of Molecular Mechanics and PM3 (semiempirical) programs four reactions involving bimolecular aromatic substitution are analyzed and their energetic and stereochemical results are compared with the average reaction rates of the experimental reactions.

The energetic analysis approaches the free energies corresponding activation and enables us to determine a reactivity scale for the studied reactions as well as for the nucleophilic and electrophilic reactives relative scale involved in their first part.

The energetic results are compared with the modeling determinations of the effect of the steric impediment related to the investigated reactives and intermediates.

Results for these comparations are correlationed and interpreted using organic chemistry basic mechanism criteria.

a* Instituto de Química Básica y Aplicada (IQBA) - Centro de Investigaciones Enseñanza Experimental (CICEE)

- Facultad de Ciencias Exactas, Químicas y Naturales (FCEQyN) - Universidad de Morón. Cabildo 134, Morón

(1708) Prov. Buenos Aires, República (54-11) 5627-2000. jorgecappetta@yahoo.com
\end{abstract}


Key Words: Nucleophilic aromatic bimolecular substitution, bivalent sulphur organic compounds, computational organic chemistry, compared reactivity, modeling of the interaction between the substrate and the nucleophile, steric impediment.

\section{INTRODUCCIÓN}

La química orgánica del azufre bivalente de aniones tiocarboxílicos, tioéteres, tioles, tiofenoles y tioésteres ${ }^{1}$ abarca reacciones, algunas de las cuales ocurren en los seres vivientes o están con ellos relacionadas estructural y/o metabólicamente. Entre las mencionadas, muchas son de gran importancia en bioenergética, además de participar en el mecanismo de acción de importantes enzimas y coenzimas ${ }^{2-4}$.

Las moléculas cuya reactividad estudiamos comparativamente en este trabajo pertenecen a algunas de las mencionadas familias de compuestos o presentan grupos funcionales análogos donde el oxígeno reemplaza al azufre. Entendemos que estas moléculas podrían ser de utilidad como "compuestos modelo", para extrapolar datos proporcionados por su estudio a casos más complejos que involucran también mecanismos bioorgánicos ${ }^{5}$.

Nos hemos propuesto encarar computacionalmente, en este trabajo, la primera etapa de la reacción general de la figura 1, comparando la reactividad de las siguientes cuatro combinaciones:

\section{Presentación general de las cuatro reacciones}

(I) Bis-(2,4-dinitrofenil) tioéter con anión tioacetato.

(II) Bis-(2,4-dinitrofenil) tioéter con anión tiobenzoato.

(III) Bis-(2,4-dinitrofenil) éter con anión tioacetato.

(IV) Bis-(2,4-dinitrofenil) éter con anión tiobenzoato.

Abreviaturas utilizadas:

Bis-(2,4-dinitrofenil)-sulfuro $=$ Sulfuro $=\varnothing 2 S$

Bis-(2,4-dinitrofenil)-éter $=$ Éter $=\varnothing 2 O$

Anión Tioacetato $=\mathrm{TA}(-)$

Anión Tiobenzoato $=\mathrm{TB}(-)$

HyperChem = Hyp.

Gaussian $=$ Gau.

Intermedio de Meisenheimer $=$ I.M.

Y además, nuestro propósito es comparar los resultados obtenidos entre sí y cotejarlos con las velocidades medias que fueron determinadas por nosotros en un trabajo anterior publicado a los efectos de verificar, si las metodologías computacionales sencillas empleadas por nosotros pueden adaptarse para la realización de estudios de reactividad comparada de sistemas análogos a los aquí investigados. 


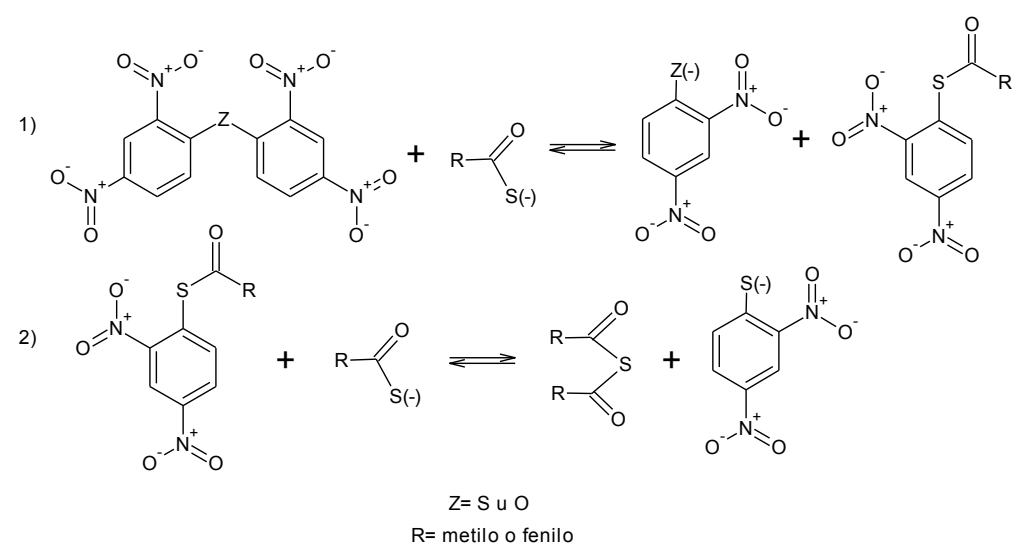

Figura 1. Formulación general de las dos etapas de las cuatro reacciones (I, II. III y IV)

Durante el desarrollo del trabajo experimental ya citado ${ }^{6}$ hemos podido establecer el orden de reactividad relativa del bis-(2,4-dinitrofenil) éter y del bis-(2,4-dinitrofenil) tioéter, frente a los aniones tioacetato y tiobenzoato en metanol-acetona (1:1), siguiendo el desarrollo de dichas reacciones por HPLC contra testigos. Los aniones tioacetato y tiobenzoato actúan como reactivos nucleofílicos frente a ambos sustratos, atacándolos mediante un mecanismo de sustitución nucleofílica aromática bimolecular.

Las velocidades medias experimentales observadas son las reportadas en tabla 1:

Tabla 1. Velocidades medias experimentales

\begin{tabular}{cc}
\hline REACCIÓN & $\mathrm{V}_{\text {media }} \times 10^{-7}(\mathrm{M} / \mathrm{min}$.) \\
(I) $\emptyset_{2} \mathrm{~S}+\mathrm{TA}(-)$ & 31,00 \\
(II) $\varnothing_{2} \mathrm{~S}+\mathrm{TB}(-)$ & 17,70 \\
(III) $\varnothing_{2} \mathrm{O}+\mathrm{TA}(-)$ & 4,44 \\
(IV) $\varnothing_{2} \mathrm{O}+\mathrm{TB}(-)$ & 0 \\
\hline
\end{tabular}

Las velocidades medias se calcularon a partir de las curvas de concentración de los reactivos sulfuro y éter versus tiempo (entre 0 y 22,5 minutos) 6 .

En el trabajo que desarrollamos a continuación se cotejan estos datos con resultados energéticos y estereoquímicos obtenidos mediante metodologías computacionales, empleando los programas Mecánica Molecular y PM3 (semiempírico) ${ }^{7-9}$.

Se fundamenta a continuación la elección de los mencionados programas. 
Se observó que al optimizar las estructuras de algunos intermedios de Meisenheimer hay problemas de deformación del enlace C-S y se detectaron también problemas de hipervalencia en el azufre, por ejemplo cuando se emplearon cálculos DFT con base 3-21g* y con bases más altas como 6-31+g(d). Por ello se eligió el programa PM3 del software Gaussian 09W e HyperChem, a fin de comparar los resultados obtenidos entre ellos, resultando además este programa consistente con los datos experimentales: se respeta el orden de los "escalones" (si bien no las distancias relativas).

También se trabajó previamente con el programa PM6 (que es superior al programa PM3) del software Gaussian09W, no obteniéndose mejores resultados que al aplicar el programa PM3 del mismo software Gaussian 09W, por tal razón elegimos este último.

\section{METODOLOGÍA}

\section{Software utilizado:}

- HyperChem Release 8.0.10 (para Windows)

- Gaussian 09W Revisión - C.01 (para Windows)

- Gauss View 5.0.9 (editor gráfico del programa Gaussian 09W

\section{Modelización}

En primera instancia se realizó el modelado molecular de todas las estructuras involucradas en la primera etapa de reacción, es decir, la estructura de los sustratos, reactivos, intermedios de reacción y productos.

Para ello se optimizó, inicialmente, modelos y energías por medio del programa mecánica molecular MM+ y luego se optimizó con el programa semiempírico PM3 del software HyperChem. Se realizó el ajuste del tipo de átomos en cada estructura, según los conocimientos teóricos de química orgánica y se tomó en cuenta la misma precisión para obtener el modelado de todas las estructuras.

Al optimizar las moléculas con MM+ y luego con PM3 se obtuvieron los resultados que se observan en la tabla 2. Los valores de energías de cada molécula están expresados en $\mathrm{kcal} / \mathrm{mol}$, utilizando un gradiente $=1 \times 10^{-3}$ para los cálculos con el software HyperChem y un gradiente que usualmente oscila entre 2,0 a 9,0 × $10^{-9}$ para los cálculos realizados con el software Gaussian.

Por medio del método semiempírico PM3 (en el software HyperChem) utilizando como estructura inicial la molécula ya optimizada por $\mathrm{MM}+$, se obtiene como resultado: la energía de unión de estas moléculas. Esta energía se calcula haciendo la diferencia entre la energía total que se obtiene con el método utilizado y la energía de los átomos aislados infinitamente separados.

El mismo proceso de optimización inicial (MM+ y luego PM3) se realizó paralelamente con el software Gaussian 09W para obtener valores simulados de energía mejor ajustados, que 
además permitieron evaluar los efectos de solvatación en las comparaciones efectuadas. Cuando se analizaron efectos de impedimento estérico, determinando distancias interatómicas con fines comparativos, se usó el programa $\mathrm{MM}+$, puesto que pone más en evidencia los mencionados efectos.

\section{RESULTADOS Y DISCUSIÓN}

Presentación de modelos y tablas

Modelos de sustratos, nucleófilos e intermedios de Meisenheimer en fase gaseosa con PM3 (Hyp.)

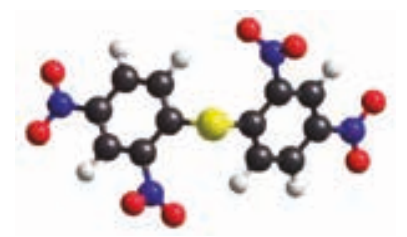

Bis-(2.4-dinitrofenil)-sulfuro

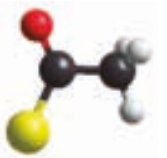

Anión tioacetato

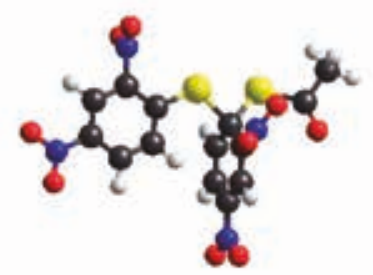

I.M. sulfuro-tioacetato

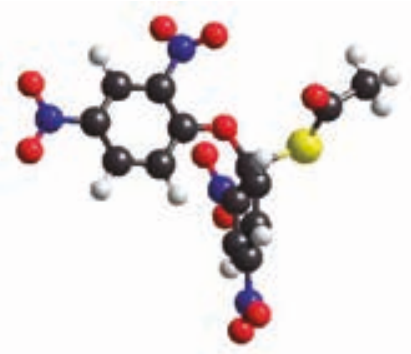

I.M. éter-tioacetato

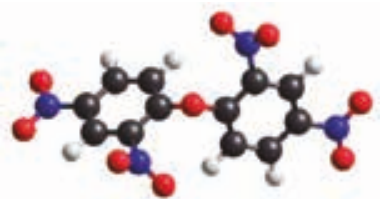

Bis-(2.4-dinitrofenil)-éter

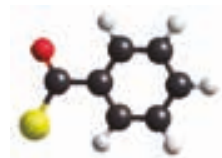

Anión tiobenzoato

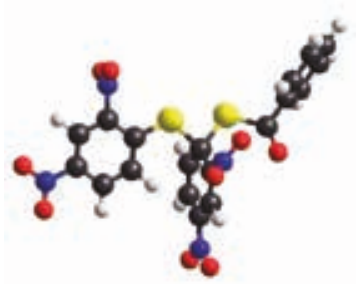

I.M. sulfuro-tiobenzoato

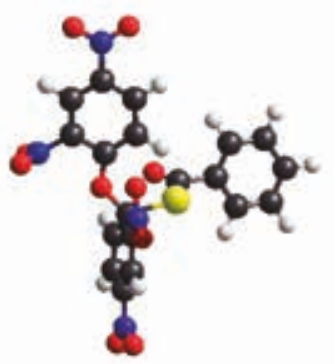

I.M. éter-tiobenzoato 
Tabla 2. Energías calculadas ( $\mathrm{kcal} / \mathrm{mol})$.

\begin{tabular}{ccccc}
\hline $\begin{array}{c}\text { REACTIVOS E } \\
\text { INTERMEDIOS }\end{array}$ & $\begin{array}{c}\text { E(MM) Hyp. } \\
\text { (fase gas.) }\end{array}$ & $\begin{array}{c}\text { E(PM3) Hyp. } \\
\text { (fase gas. ) }\end{array}$ & $\begin{array}{c}\text { E(PM3) Gau. } \\
\text { (fase gas. ) }\end{array}$ & $\begin{array}{c}\text { E(PM3) Gau. } \\
\text { (metanol) }\end{array}$ \\
$\begin{array}{c}\text { Eulfuro } \\
\text { Éter }\end{array}$ & 14,12 & $-3311,05$ & 47,38 & 22,06 \\
Anión Tioacetato & 10,26 & $-3351,73$ & $-1,34$ & $-27,53$ \\
Anión Tiobenzoato & 1,45 & $-693,75$ & $-69,77$ & $-134,83$ \\
I.M. Sulfuro-tioacetato & 5,19 & $-1620,13$ & $-37,78$ & $-103,31$ \\
I.M. Sulfuro-tiobenzoato & 15,46 & $-4048,98$ & $-65,59$ & $-118,02$ \\
I.M. Éter-tioacetato & 21,50 & $-4972,27$ & $-31,03$ & $-82,17$ \\
I.M. Éter-tiobenzoato & 17,90 & $-4085,92$ & $-111,06$ & $-163,23$ \\
\hline
\end{tabular}

E(MM): Energía estérica de la molécula (energía potencial estérica), expresada en $\mathrm{kcal} / \mathrm{mol}$. E(PM3) HyperChem: Sumatoria de la energía electrónica total, denominada "energía de unión", expresada en $\mathrm{kcal} / \mathrm{mol}$.

E(PM3) Gaussian: Sumatoria de la energía electrónica total y la repulsión entre los núcleos en la molécula, expresada en kcal/mol. No es igual a la energía PM3 del programa HyperChem

Tabla 3. Comparativa de variaciones de energías cotejadas con velocidades medias experimentales.

\begin{tabular}{cccc}
\hline REACCIÓN & $\begin{array}{c}\mathrm{V}_{\text {media }} \times 10^{-7}(\mathrm{M} / \mathrm{min} .) \\
\text { (metanol-acetona) }\end{array}$ & $\begin{array}{c}\Delta \mathrm{E}(\mathrm{PM} 3) \mathrm{Gau} . \\
\text { (metanol) } \\
\mathrm{kcal} / \mathrm{mol}\end{array}$ & $\begin{array}{c}\Delta \mathrm{E}(\mathrm{PM} 3) \mathrm{Gau} . \\
\text { (fase gas. } \\
\mathrm{kcal} / \mathrm{mol}\end{array}$ \\
(I) $\varnothing_{2} \mathrm{~S}+\mathrm{TA}(-)$ & 31,00 & $-5,25$ & $-43,19$ \\
(II) $\emptyset_{2} \mathrm{~S}+\mathrm{TB}(-)$ & 17,70 & $-0,91$ & $-40,63$ \\
(III) $\varnothing_{2} \mathrm{O}+\mathrm{TA}(-)$ & 4,44 & $-0,87$ & $-39,95$ \\
(IV) $\varnothing_{2} \mathrm{O}+\mathrm{TB}(-)$ & 0 & 1,89 & $-36,43$ \\
\hline
\end{tabular}

I.M.: Intermedio de Meisenheimer.

$\Delta \mathrm{E}(\mathrm{PM})=\mathrm{E}$ I.M. $-\sum \mathrm{E}$ Reactivos, es la variación de energía PM3 entre el I.M. y la suma de los reactivos, que entendemos se aproxima a la energía de activación correspondiente a la primera etapa de cada reacción, en la hipótesis que la energía del I.M. pueda confundirse con la energía del estado de transición de la etapa limitante. 
Tabla 4. Comparativa de reactividades promedio (permite evaluar nucleofilias y electrofilias relativas de aniones y sustratos).

\begin{tabular}{cccc}
\hline PROMEDIOS & $\begin{array}{c}\mathrm{V}_{\text {media }} \text { 10-7 (M/min.) } \\
\text { (metanol-acetona) }\end{array}$ & $\begin{array}{c}\Delta \mathrm{E}(\mathrm{PM} 3) \mathrm{Gau} . \\
\text { (metanol) } \\
\mathrm{kcal} / \mathrm{mol}\end{array}$ & $\begin{array}{c}\Delta \mathrm{E}(\mathrm{PM} 3) \mathrm{Gau} . \\
\text { (fase gas. }) \\
\mathrm{kcal} / \mathrm{mol}\end{array}$ \\
$(\mathrm{I})+(\mathrm{II}) / 2$ & 24,35 & $-3,08$ & $-41,91$ \\
$(\mathrm{III})+(\mathrm{IV}) / 2$ & 2,22 & 0,51 & $-38,19$ \\
$(\mathrm{I})+(\mathrm{III}) / 2$ & 17,72 & $-3,06$ & $-41,57$ \\
$(\mathrm{II})+(\mathrm{IV}) / 2$ & 8,85 & 0,49 & $-38,53$ \\
\hline
\end{tabular}

Se han cotejado e interpretado los datos cinéticos experimentales con los energéticos obtenidos computacionalmente arriba tabulados, acorde con los siguientes criterios:

- La primera etapa de cada reacción considerada no es significativamente influenciada en su desarrollo cinético por la segunda ${ }^{6}$.

- El mecanismo común, subyacente a las primeras etapas que comparamos es de sustitución nucleofílica aromática bimolecular ${ }^{10-13}$ que generalmente, permite confundir la energía del intermedio de Meisenheimer (I.M.) con la energía del estado de transición que lo antecede, como hacemos aquí.

- Se asume que las velocidades medias determinadas experimentalmente que reportamos puedan equipararse en primera aproximación a las correspondientes constantes cinéticas de la primera etapa de cada reacción a los fines comparativos, hallándose además estas últimas en relación exponencial inversa con las energías libres de Gibbs de activación estándar molar $\left(\Delta \mathrm{G}^{0} \neq\right)^{15}$, acorde con la formulación termodinámica del estado de transición.

- $\quad$ Las variaciones de energía: $\Delta \mathrm{E}(\mathrm{PM} 3)=\mathrm{E}$ I.M. $-\sum \mathrm{E}$ Reactivos, calculadas mediante los software HyperChem y Gaussian ${ }^{11,12}$ se asimilan a las correspondientes variaciones

- de energía interna de activación estándar molar $\left(\Delta \mathrm{U}_{0} \neq\right)$.

- Además, se consideran los valores $\Delta \mathrm{U}_{0} \neq$ comparables con los correspondientes valores

- $\Delta \mathrm{H}_{0} \neq$ (entalpía de activación estándar molar), en solución y en fase gaseosa, considerando en este último caso que $\Delta \mathrm{H}_{0} \neq=\Delta \mathrm{U}_{0} \neq+\mathrm{RT} \Delta \mathrm{n}(\mathrm{g})$, donde $\Delta \mathrm{n}$ (gas) es, en los casos por nosotros considerados, siempre uno ${ }^{16}$.

- Es decir que, en las comparaciones entre las primeras etapas los valores de $\Delta \mathrm{H} 0 \neq \mathrm{y}$ $\Delta \mathrm{U} 0 \neq$ difieren por la misma constante.

- Asumimos entonces que, siempre en primera aproximación, resulta ser:

- $\Delta \mathrm{G}_{0} \neq=\Delta \mathrm{H}_{0} \neq-\mathrm{T} \Delta \mathrm{S}_{0} \neq=\Delta \mathrm{A}_{0} \neq=\Delta \mathrm{U}_{0} \neq-\mathrm{T} \Delta \mathrm{S}_{0} \neq$, siendo $\Delta \mathrm{A}_{0} \neq$ la energía libre de activación de Helmholtz estándar molar ${ }^{16}$.

- Entendemos que la variación $\Delta \mathrm{S}_{0} \neq$ aquí considerada es igual en los cuatro casos que comparamos en este trabajo en cuanto es la que se halla asociada a la variación de grados de libertad vinculados con $\Delta \mathrm{n}$.

- La variación de entropía asociada a la variación de grados de libertad, propios de las estructuras moleculares e iónicas, involucradas en la transición desde los reactivos hasta el correspondiente intermedio de Meisenheimer, se analizarán separadamente en 
este trabajo, aproximando las mismas mediante el uso de criterios estereoquímicos de impedimento estérico fundamentados en la determinación y comparación de distancias interatómicas.

\section{Comparación e interpretación de los valores computacionales y experimentales calculados}

El análisis de la tabla 3 indica que las variaciones de energía calculadas en fase gaseosa y en metanol presentan un ordenamiento que se corresponde con el presentado por las velocidades medias determinadas experimentalmente en metanol-acetona $(1: 1)$ :

$$
\text { I }>\text { II }>\text { III }>\text { IV }
$$

generando el solvente polar prótico un efecto de disminución general de reactividad pero respetando el orden tal como fuera calculado en fase gaseosa.

Este hecho es relacionable con la fuerte solvatación sufrida por los aniones en solvente polar prótico.

Analizando la tabla 4 y comparando, en cada una de las tres columnas, el promedio de los valores correspondientes a las reacciones I y II con el análogo promedio referido a las reacciones III y IV, se infiere que el sulfuro es mejor sustrato que el éter frente a ambos aniones, en el contexto de las mismas.

Razonando análogamente con los promedios de I y III, y de II y IV, se concluye que el anión tioacetato es mejor nucleófilo que el anión tiobenzoato frente a ambos sustratos, en el correspondiente contexto.

Volviendo a analizar la tabla 3 en función de los datos presentados en la tabla 4 se observa que el orden teórico resulta ser en todos los casos acorde con el orden experimental.

Cabe resaltar también que en la reacción I convergen el mejor nucleófilo y el mejor electrófilo, y en la IV el peor nucleófilo y el peor electrófilo; mientras en las II y III la convergencia es mixta y la II prevalece sobre la III. Lo que está indicando que los nucleófilos solo influyen secundariamente en el orden de reactividad global I > II > III > IV ya establecido; y que es en las reacciones II y III donde mejor se evidencia, aunque sea secundariamente, el efecto de los mismos.

Datos de la tabla 3 indican que son los electrofílicos los principales condicionantes del orden de reactividad presentado por las cuatro reacciones que comparamos, en efecto: la mayor reactividad se asocia a las reacciones I y II, respecto a las III y IV).

Todo lo afirmado vale en fase gaseosa, en metanol (estudio teórico) y en metanol-acetona (estudio experimental).

El método de la resonancia, cualitativamente aplicado, da cuenta de la mayor estabilidad (menor reactividad) del éter cuando se la compara con la del sulfuro. En efecto, la conjugación $\mathrm{C}$ aromático-oxígeno respecto a la $\mathrm{C}$ aromático-azufre es más efectiva (téngase en cuenta la diferencia de volumen atómico entre oxígeno y azufre).

Además, la mayor estabilidad del anión tiobenzoato respecto al anión tioacetato (resonancia 
del grupo fenilo, mientras que el grupo metilo solo hiperconjuga) avala la mayor reactividad del anión tioacetato.

Considerando en general el efecto del solvente polar y prótico (aunque la proticidad resulte disminuida en la mezcla solvente utilizada experimentalmente respecto al metanol, usado para el análisis computacional), podemos concluir que:

- En las cuatro reacciones comparadas el solvente produce una disminución general de reactividad pero sin alterar el orden de la misma: I $>$ II $>$ III $>$ IV.

- También respeta el orden de nucleofilia y electrofilia relativos, como se estableció en fase gaseosa.

\section{Análisis e interpretación de los principales efectos estéricos "en fase gaseosa", involucrados en la primera etapa de las cuatro reacciones aquí estudiadas}

Tabla 5. Distancias principales asociadas al "efecto orto cercano" ( $\AA$ ) con MM+ (Hyp.).

\begin{tabular}{cccc}
\hline SUSTRATOS E I.M. & N(-)io - Ho & O(-)ico - Ho & O(-)ilo - Ho \\
$\emptyset_{2} \mathrm{~S}$ & 5,120 & 5,029 & 6,385 \\
$\emptyset_{2} \mathrm{O}$ & 3,550 & 2,964 & 4,832 \\
$(\mathrm{I})$ & 3,629 & 3,473 & 4,900 \\
$(\mathrm{II})$ & 3,538 & 3,843 & 4,453 \\
(III) & 3,300 & 3,829 & 4,078 \\
(IV) & 4,513 & 3,679 & 5,734 \\
\hline
\end{tabular}

N(-)io - Ho: distancia entre el No (unido al anillo con carga (-) que está a la izquierda en el esquema) y el Ho del otro anillo.

$\mathrm{O}(-)$ ico - Ho: distancia entre el O cercano unido al No (unido al anillo con carga (-) que está a la izquierda en el esquema) y el Ho del otro anillo.

$\mathrm{O}(-)$ ico - Ho: distancia entre el O lejano unido al No (unido al anillo con carga (-) que está a la izquierda en el esquema) y el Ho del otro anillo. (ver figura2).

Tabla 6. Distancias principales asociadas al "efecto orto lejano" ( $\AA$ ) con MM+ (Hyp.).

\begin{tabular}{cccc}
\hline SUSTRATOS E I.M. & Ndo - H(-)io & Odco - H(-)io & Odlo - H(-)io \\
$\emptyset_{2} \mathrm{~S}$ & 5,120 & 5,029 & 6,385 \\
$\emptyset_{2} \mathrm{O}$ & 3,550 & 2,964 & 4,832 \\
$(\mathrm{I})$ & 5,426 & 5,044 & 6,684 \\
$(\mathrm{II})$ & 5,382 & 5,861 & 6,012 \\
(III) & 4,668 & 5,053 & 5,350 \\
(IV) & 2,900 & 3,195 & 3,576 \\
\hline
\end{tabular}


Ndo - H(-)io: distancia entre el No (unido al anillo sin carga que está a la derecha en el esquema) y el $\mathrm{H}(-)$ io unido anillo con carga (-).

Odco - H(-)io: distancia entre el $\mathrm{O}$ cercano unido al No (unido al anillo sin carga que está a la derecha en el esquema) y el H(-)io unido al anillo con carga (-).

Odlo - Ho: distancia entre el O lejano unido al No (unido al anillo sin carga que está a la derecha en el esquema) y el H(-)io anillo con carga (-). (ver figura 3).

\section{$\mathrm{Z}=\mathrm{S}$ u $\mathrm{O}$ \\ $\mathrm{R}=$ metilo o Enilo}

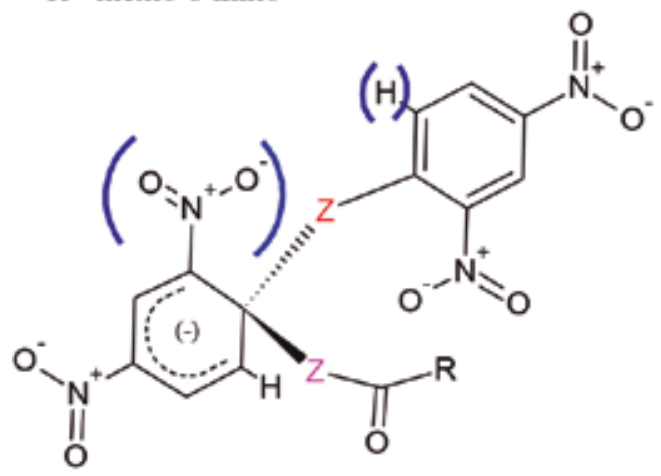

Figura 2. Formulación indicante el efecto orto cercano en un I.M. tipo (ver los paréntesis).

$$
\begin{aligned}
& \mathrm{Z}=\mathrm{S} \text { u O } \\
& \mathrm{R}=\text { metilo o fenilo }
\end{aligned}
$$

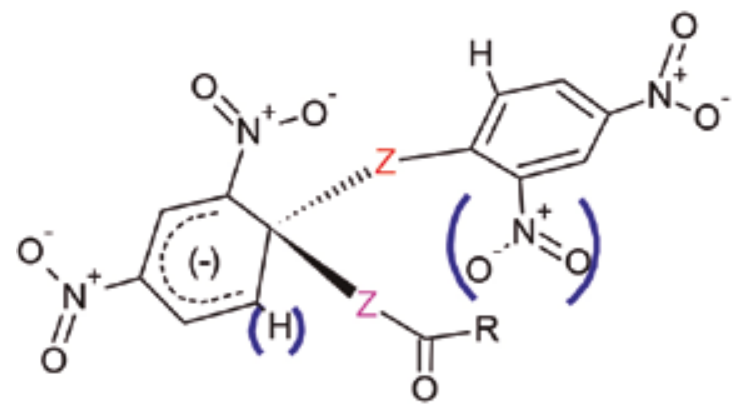

Figura 3. Formulación indicante el efecto orto lejano en un I.M. tipo (ver los paréntesis). 
Tabla 7. Comparativa y abarcante que presenta (fila por fila) el promedio de las distancias $(\AA)$ del efecto orto cercano (columna A) y lejano (columna B) y su promediación final en la columna C.

\begin{tabular}{|c|c|c|c|}
\hline & COLUMNA (A) & COLUMNA (B) & COLUMNA $(\mathrm{C})$ \\
\hline $\begin{array}{c}\text { SUSTRATOS } \\
\text { E I.M. }\end{array}$ & $\sum_{\text {orto cercanas }) / 3}$ & $\begin{array}{c}\sum(\text { distancias } \\
\text { orto lejanas }) / 3\end{array}$ & $\begin{array}{c}\text { Promedio filas de } \\
\text { las columnas (A) } \\
\text { y (B) }\end{array}$ \\
\hline$\varnothing_{2} \mathrm{~S}$ & 5,510 & 5,510 & 5,510 \\
\hline$\varnothing_{2} \mathrm{O}$ & 3,782 & 3,782 & 3,782 \\
\hline (I) & 4,000 & 5,718 & 4,859 \\
\hline (II) & 3,940 & 5,750 & 4,845 \\
\hline (III) & 3,730 & 5,020 & 4,375 \\
\hline (IV) & 4,642 & 3,220 & 3,933 \\
\hline
\end{tabular}

Columna (A): Promedio de los datos de la tabla 5. Columna (B): Promedio de los datos de la tabla 6.

Los promedios de distancias obtenidos efectuando la lectura horizontal de las tablas 5 y 6 fueron reportados en las columnas A y B de la tabla 7 y se corresponden con lo denominado aquí como "efecto orto cercano y lejano". Estos promedios fueron a su vez promediados y los valores finales aparecen ordenados en la columna $\mathrm{C}$ de la misma tabla $\mathrm{y}$ son los indicadores del "efecto de jaula" que se genera en el entorno donde se encuentran los carbonos electrofílicos $\mathrm{C} 1$ y C'1, sea del éter como del sulfuro, interaccionantes con el azufre nucleofílico y covalentemente unidos a éste en el correspondiente intermedio de Meisenheimer. Relacionamos así el "efecto de jaula" con el concepto de impedimento estérico en el mencionado entorno.

\section{Impedimento estérico comparado presente en éter y sulfuro}

En primer lugar se observa que las dos distancias (orto nitro- $\mathrm{H}$ orto) (columnas A y B de la tabla 7) son iguales entre sí en el éter y respectivamente también lo son en el sulfuro, pero mayores en el sulfuro. Por lo tanto en este análisis no hace falta distinguir entre "efecto orto cercano y lejano".

Además, el impedimento estérico que presenta el éter respecto al sulfuro queda puesto en evidencia en la tabla 6 donde los oxígenos del grupo nitro en $\mathrm{C}_{2}$ con el hidrógeno en $\mathrm{C}_{6}{ }_{6} \mathrm{y}$ los oxígenos del grupo nitro en $\mathrm{C}_{2}{ }_{2}$ con el hidrógeno en $\mathrm{C}_{6}$ se hallan más cerca en el primer sustrato que en el segundo.

En efecto, el promedio de las distancias de los átomos constituyentes del grupo orto nitro de un anillo respecto al hidrógeno en orto del otro anillo es de 5,510 ^ para el sulfuro y de 3,782 Å para el éter. 
Esto, sugiere que los carbonos 1 y 1', se hallan estéricamente más protegidos en el éter que en el sulfuro, frente al ataque del nucleófilo, quedando un anillo más rotado respecto al otro en el éter, lo que lleva a que los carbonos electrofílicos resulten más desprotegidos en el sulfuro. A los efectos, téngase en cuenta también que la distancia $C_{1}-C_{1}$ ' en el sulfuro es 3,190 Å y en el éter es 2,387 $\AA$, lo que también favorece la mencionada rotación para el éter, por el mayor impedimento provocado por los grupos en orto antes mencionado.

Cabe destacar además (allí hasta donde el impedimento estérico influye) que el estudio aquí expuesto presenta, en lo que hace a los sustratos éter y sulfuro, buena concordancia con hechos experimentales anteriormente determinados por nosotros ${ }^{6,14}$.

Haciendo ahora referencia a los nucleófilos el anión tioacetato, poseyendo la misma carga que el anión tiobenzoato y menor volumen donde ésta se distribuye, resulta más reactivo que el anión aromático.

\section{Impedimento estérico parcial que se presenta y compara en los intermedios de Meisenheimer de la primera etapa de las reacciones aquí investigadas}

El análisis de los valores representados en la columna $\mathrm{C}$ de la tabla 7 , indica que el apiñamiento estérico en los intermedios de Meisenheimer se presenta en el orden:

IV $>$ III $>$ II $>$ I, asociable a tensionamientos cada vez mayores de izquierda a derecha, que se corresponden bien con las variaciones de energía que determinamos y comparamos.

El impedimento estérico pudo así analizarse separadamente del contexto energético que los abarca, con el objetivo de mejor evidenciar su aporte a las variaciones energéticas en cuestión, más allá de que sea determinante o solamente contribuyente a las mismas.

Por último, cabe resaltar que el análisis estereoquímico realizado (además de resultar limitado por la restringida elección de las distancias comparadas para su determinación) considera separadamente el impedimento estérico de los reactivos y de los intermedios de Meisenheimer correspondientes, pero no la variación espacial progresiva entre los átomos de los reactivos que se reacomodan a lo largo de la coordenada de reacción hasta alcanzar la conformación espacial del estado de transición o de un casi equivalente intermedio de Meisenheimer.

Igualmente, el mencionado análisis ayuda a mejor comprender las causas de las diferencias de reactividad analizadas durante este estudio.

\section{CONCLUSIONES}

- Los programas computacionales empleados permitieron investigar las cuatro reacciones objeto de nuestro estudio, comparando mediante técnicas de simulación la primera etapa de las mismas en su desarrollo energético y cotejar los resultados así obtenidos con los 
datos de velocidades medias experimentalmente determinadas en un trabajo anterior ${ }^{6}$. El orden de reactividades resultó ser en ambos casos: I > II > III > IV, ejerciendo el solvente polar prótico una disminución general de reactividad, pero sin alterar el mencionado orden.

- De los nucleófilos involucrados el anión tioacetato se presenta como el mejor nucleófilo en el contexto de las reacciones comparadas y el sulfuro como el mejor electrófilo.

- Los efectos estéricos analizados en fase gaseosa, separados de los valores energéticos que los engloban, indican que el éter resulta ser más impedido estéricamente en el entorno susceptible de ataque nucleofílico que el sulfuro correspondiente. Además, el impedimento estérico relativo estimado para los cuatro intermedios de Meisenheimer se correlaciona con el orden de reactividad por nosotros propuesto energética y experimentalmente, aun sin ser el único causante del mismo.

- Cabe resaltar que gran parte de los hechos expuestos y analizados en este trabajo los hemos descrito e interpretado haciendo uso de conceptos pertenecientes a la Química Orgánica básica, lo que abre las puertas para analizar la posibilidad de adaptar programas, modelos y criterios interpretativos relativamente sencillos para incorporarlos en el ámbito educacional (nivel terciario y universitario) al desarrollo de cursos de introducción a la Química Orgánica Computacional básica, aplicada al estudio comparativo y semicuantitativo de reacciones semejantes a las aquí investigadas que presenten el mismo mecanismo subyacente.

- Además, entendemos que criterios similares a los aquí desarrollados podrían ser útiles en el ámbito de la investigación química básica y/o aplicada, para delinear estrategias en la comparación de reactividades haciendo uso de programas computacionales sencillos antes de acudir a otros más complejos o de implementar metodologías a nivel experimental que permitan un adecuado chequeo, pero que podrían requerir un alto costo en reactivos, equipamiento y mayores tiempos operativos.

\section{AGRADECIMIENTOS}

Al Sr. Decano de la Facultad de Ciencias Exactas Químicas y Naturales de la Universidad de Morón: Dr. Aquiles Ferranti, por la confianza otorgada a nuestras investigaciones.

Al Dr. David Kuczynski, por la asidua colaboración prestada cuando se desempeñó como Director de Investigaciones de la Facultad y que actualmente continúa prestándonos.

A la Dra. Ethel Coscarello, por habernos iniciado en la metodología de la Química Computacional, en los albores del esfuerzo por nosotros realizado al desarrollar este trabajo. 


\section{REFERENCIAS BIBLIOGRÁFICAS}

1. Patai S. The chemistry of the thiol group, Part I y II. New York: John Wiley \& Sons. 1974. p.81-86, 125- 131 .

2. Fox MA. Whitesell, J. K. Química Orgánica. 2 2a edición. México DF: Ed. Pearson; 2000. Cap. 20 y 22.

3. Voet D, Voet JG. Bioquímica. Parte III, $3^{\text {ra }}$ edición. Buenos Aires: Ed. Medica Panamericana; 2006. p. 471-562.

4. Bruice TC, Benkovic SJ. Bioorganic Mechanisms. Vol. I. New York: Ed. Benjamin WA; 1966. Cap I y III.

5. Micheli CA. Introducción a los mecanismos bioorgánicos. Morón: Ed. Praia; 2015. p. 22-23.

6. Gallo IM, Caresana AM, Cappetta JE, Micheli CA. Reactividad del bis-(2,4-dinitrofenil)éter y del análogo tioéter, frente a aniones carboxílicos y tiocarboxílicos. Rev Soc Quím Perú. 2012; 78 (2): 91-104.

7. Foresman JB, Frisch A. Exploring Chemistry with Electronic Structure Methods. $2^{\text {nd }}$ edition. Pittsburgh: Gaussian; 1996. p.208-211.

8. Bachrach SM. Computational Organic Chemistry. New York: John Wiley \& Sons; 2007. p.373-391.

9. Rogers, D.W. Computational Chemistry using the PC. $3^{\text {rd }}$ Edition. New York: John Wiley \& Sons; 2003. p.93-130; 279-298.

10. Bunnett JF, Zahler RE. Aromatic Nucleophilic Substitution Reactions. Chem. Rev. 1951; 49 (2): 273-412.

11. Bunnett JF, Meritt WD. The nucleophilic reactivity of sodium thiophenoxide with aromatic substrates. J Am Chem Soc. 1957; 79 (22): 5967-5969.

12. Reinheimer JD, Hostetler W. The aromatic nucleophilic substitution reaction: the effect of initial concentration on the rate of the reaction of $\mathrm{MOH}$ on 2,4 dinitrohalobenzenes. Ohio J Sc. 1964; 64 (4): 275-283.

13. Guanti G, Dell'Erba C, Thea S, Leandri G. Reactivity of thiophenoxide ion toward oand p-halogenonitrobenzenes. J Chem Soc Perkin Trans 2. 1975: 389-391.

14. Wright V; Gallo I, Gonzalez P, Sciarrotta E, Micheli CA. Introducción al estudio de la reactividad del bis-(2,4-dinitrofenil)- éter y del bis-(2,4-dinitrofenil)- tioéter frente al anión metóxido en acetona. Quím Nat. 2010; 8: 35-50.

15. Chang R. Fisicoquímica para las Ciencias Químicas y Biológicas, $3^{\text {ra }}$ Edición. México DF: Mc Graw-Hill, Interamericana; 2008. Cap. 12, p. 475-479.

16. Waser J. Termodinámica Química Fundamental. Baecelona: Ed. Reverté; 1972. Cap. 2 y 5, p.48-49,104-107. 